\title{
The Spanish Infrared Camera onboard the EUSO-BALLOON (CNES) flight on August 24, 2014
}

M.D. Rodríguez Frías ${ }^{* 1,2,3}$, J. Licandro ${ }^{4}$, M.D. Sabau ${ }^{5}$, J.L. Sánchez ${ }^{6}$, S. Franchini ${ }^{7}$, L. López ${ }^{6}$, L. Ramírez ${ }^{8}$, E. Joven ${ }^{4}$, M. Reyes $^{4}$, C. González-Alvarado ${ }^{5}$, T. Belenguer ${ }^{5}$, J. Meseguer ${ }^{7}$, I. Pérez-Grande ${ }^{7}$, G. Sáez-Cano ${ }^{1}$, J. F. Soriano ${ }^{1}$, J. H-Carretero' ${ }^{1}$, H. Prieto $^{1}$, J.A. Morales de los Ríos ${ }^{1}$, Y. Martín ${ }^{4}$, A. Merino ${ }^{6}$, M. Sanz-Palomino ${ }^{5}$, E. García-Ortega $^{6}$, E. Gascón ${ }^{6}$, S. Fernández-González ${ }^{6}$, G. Alonso ${ }^{7}$, E. Roibas ${ }^{7}$, A. Sanz-Andrés ${ }^{7}$, S. Pindado ${ }^{7}$, O. Maroto ${ }^{9}$, L. Díez-Merino ${ }^{9}$, A. Tomás ${ }^{9}$, J. Carbonell ${ }^{9}$, C. Echeandía ${ }^{8}$, S. Pérez-Cano ${ }^{8}$ and L. del Peral ${ }^{1,3}$ for the JEM-EUSO Collaboration.

${ }^{1}$ SPace \& AStroparticle (SPAS) Group, UAH, Madrid, Spain.

${ }^{2}$ IFIC, CSIC, Universitat de València. Dpto. Física Atómica, Molecular y Nuclear, Universitat de València.

${ }^{3}$ ISDC, Astronomy Dept. University of Geneva, Switzerland.

${ }^{4}$ Instituto de Astrofísica de Canarias (IAC), Vía Láctea S/N, Tenerife, Spain.

${ }^{5}$ LINES Laboratory, Instituto Nacional de Técnica Aeroespacial (INTA), Madrid.

${ }^{6}$ GFA. IMA. University of León, León, Spain.

${ }^{7}$ IDR/UPM, E. T. S. I. Aeronáutica y del Espacio, Universidad Politécnica de Madrid, Madrid, Spain.

${ }^{8}$ ORBITAL AEROSPACE, Pol. Ind. Las Fronteras, San Fernando de Henares, Madrid, Spain.

${ }^{9}$ SENER, Parc de l'Alba, Cerdanyola del Vallès, Barcelona, Spain.

E-mail: dolores.frias@uah.es

The EUSO-Balloon (CNES) campaign was held during Summer 2014 with a launch on August 24. In the gondola, next to the Photo Detector Module (PDM), a completely isolated Infrared camera was allocated. Also, a helicopter which shooted flashers flew below the balloon. We have retrieved the Cloud Top Height (CTH) with the IR camera, and also the optical depth of the nonclear atmosphere have been inferred with two approaches: The first one is with the comparison of the brightness temperature of the cloud and the real temperature obtained after the pertinent corrections. The second one is by measuring the detected signal from the helicopter flashers by the IR Camera, considering the energy of the flashers and the location of the helicopter.

The 34th International Cosmic Ray Conference,

30 July- 6 August, 2015

The Hague, The Netherlands

\footnotetext{
* Speaker.
} 


\section{Introduction}

JEM-EUSO (Extreme Universe Space Observatory on Japanese Experiment Module) is a novel space-based experiment that will be launched in 2019. Its aim is to observe EAS (Extensive Air Showers) produced by UHECRs (Ultra High Energy Cosmic Rays) and EECRs (Extremely High Energy Cosmic Rays) in an energy range between $10^{19.5} \mathrm{eV}$ and $10^{21} \mathrm{eV}$ [1]. Observing from space (this is, with a larger observation area), a larger exposure is achievable [2]. And this is required, due to the small UHECRs flux. The arrival direction map will provide us information on the origin of the UHECRs, probably allowing us to identify the nearest UHECR sources with known astronomical objects. This will allow us to understand their acceleration mechanisms. Moreover, it will help to clarify the acceleration and emission mechanisms, and to confirm the Greisen-Zatsepin-Kuz'min suppression. JEM-EUSO will use the atmosphere as a detector [3]. Therefore, information about properties of the Earth's atmosphere and presence of clouds is highly needed [4]. The telescope includes an Atmospheric Monitoring system (AMS) which provides information on the clouds and aerosol distribution, as well as their optical properties within the telescope Field of View (FoV) [5, 6]. The AMS will consist of an infrared camera (IR), and a LIght Detection And Ranging device (LIDAR).

There are three JEM-EUSO pathfinders at different stages (either functioning or under construction): EUSO-Balloon, EUSO-TA and Mini-EUSO. The objectives of these pathfinder missions are: to perform a full scale end-to-end test of the JEM-EUSO concept and key technologies, to test the electronic components in stratospheric conditions, and to measure the UV background at high altitudes.

\section{EUSO-Balloon}

EUSO-Balloon is a balloon-borne experiment developed by the JEM-EUSO consortium [7]. Its aim is to test the technologies and methods used in the forthcoming main experiment, through a series of stratospheric balloon flights that have already started in August, 2014. EUSO-Balloon, as the main mission, is an imaging UV telescope. It points towards the nadir from an altitude of about $40 \mathrm{~km}$. It is equipped with one Photo Detector Module (PDM) identical to one of the JEM-EUSO instrument, and three Fresnel lenses which are prototypes of those which will be installed in JEMEUSO. The instrument will cover a Field of View of $12^{\circ} \times 12^{\circ}$ in a wavelength range between 290 and $430 \mathrm{~nm}$. The EUSO-Balloon, as well as the main mission, will have an Infrared Camera to analyze the atmospheric properties along the Balloon flight.

The objectives of the IR camera are:

- To validate the JEM-EUSO IR camera mission concept

- To obtain real data with microbolometer detector (used in JEM-EUSO IR camera).

- To assess the wavelength bands and filters selection.

- To validate and optimize the retrieval algorithms.

- To validate and optimize stereo vision technique. 
- To validate and assess part of calibration strategy .

- To validate and optimize temperature retrieval algorithms.

\section{EUSO-Balloon IR Camera Design}

The IR Camera is a stand-alone subsystem within the balloon, which provides images centered at $10.8 \mu \mathrm{m}$ and $12 \mu \mathrm{m}$ (medium infrared), thanks to a ULIS UL 04171 microbolometer and two filters centered in that wavelengths with $0.85 \mu \mathrm{m}$ of bandwidth. The imaging system is exactly as the JEM-EUSO IR Camera in its BreadBoard Model.

The camera module is the IRXCAM-640 developed to handle the microbolometer ULIS UL04-17-1 [8]. It incorporates a shutter control. The electronics show a very weak level of noise, which is lower than the noise level of the detector. The IRXCAM-640 software controls the detector, calibrates and characterizes the IR-camera. However, this software is only used to center the target in the FoV. Therefore, a specific software has been developed to control the IRXCAM-640.

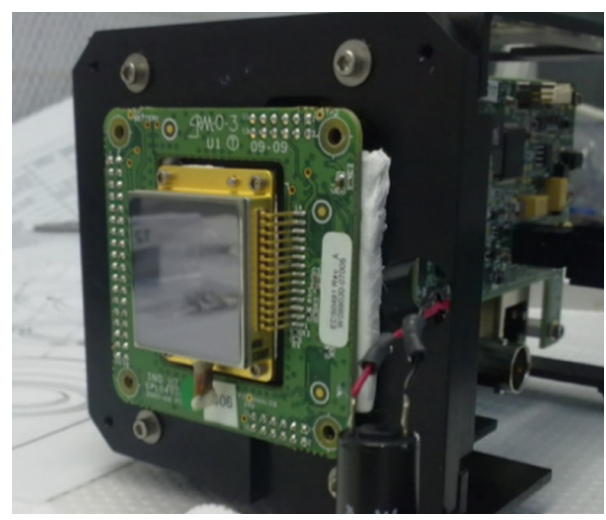

Figure 1: ULIS UL-04-17-1 microbolometer.

The ULIS detector is an infrared opto-electronic device sensitive to radiation in the long wave spectral range. It includes a microbolometer Focal Plane Array (FPA) comprised of a $640 \times 480$ pixels. The pixel pitch is $25 \mu \mathrm{m}$ by $25 \mu \mathrm{m}$, being the image size is $16 \mathrm{~mm}$ by $12 \mathrm{~mm}$. This detector array, made from silicon resistive bolometer microbridges, is connected to a silicon ReadOut Integrated Circuit (ROIC). It also includes a Thermo Electric Cooler (TEC), which is controlled by the IRXCAM-640. The detector has several internal parameters that need to be configured by the user, according to the measurement range, environment, and camera configuration. These parameters are optimized to obtain the lowest Noise Equivalent Temperature Difference (NETD) possible. Although the microbolometer could operate without TEC and improve the system efficiency (lowest power consumption), for applications which require an accurate FPA thermal stability (our case), the module provides the Focal Plane Array (FPA) temperature value that can be used to control the TEC already integrated into the FPA package. The accuracy of the temperature sensor is $10 \mathrm{mK}$ [9].

For the camera optics we decided to acquire a SURNIA Lenses equipment from the company Janos Technology. Due to its very fast F\#, the maximum amount of energy will reach our focal 
Table 1: Technical specifications of the camera module IRXCAM-640.

\begin{tabular}{c|c}
\hline & IRXCAM-640 \\
\hline \hline Sensor & $640 \times 480$ pixels \\
& ULIS UL 04 17 1 \\
& uncooled microbolometer \\
\hline Power supply & $9-12 \mathrm{~V} \mathrm{DC}$ \\
\hline Dimensions & $65 \mathrm{~mm} \mathrm{(H)}$ \\
& $59 \mathrm{~mm} \mathrm{(W)}$ \\
& $125 \mathrm{~mm}(\mathrm{~L})$ \\
\hline Weight & $250 \mathrm{~g}$ \\
\hline Temperature & Operating: -30 to $55^{\circ} \mathrm{C}$ \\
& Storage: -40 to $80^{\circ} \mathrm{C}$ \\
\hline
\end{tabular}

Table 2: Technical specifications of the $\mu$ bolometer UL 04171.

\begin{tabular}{c|c}
\hline & UL 04 17 1 \\
\hline \hline Pixel-pitch & $25 \mu \mathrm{m}$ \\
\hline Dimensions & $7.7 \mathrm{~mm} \mathrm{(H)}$ \\
& $32 \mathrm{~mm} \mathrm{(W)}$ \\
& $23.5 \mathrm{~mm}(\mathrm{~L})$ \\
\hline Weight & $<25 \mathrm{~g}$ \\
\hline Power comsumption & $<300 \mathrm{~mW}($ without TEC) \\
\hline NETD & $<120 \mathrm{mK}$ \\
\hline
\end{tabular}

plane. Although it is designed for camera systems with a stop, and longer back working distance (common for cooled systems or detectors requiring a radiation shield), it can be adapted to most longwave infrared (LWIR) cameras. Moreover, the IRXCAM-640 manufacturer has provided us the mechanical mount to adapt these lenses to the device.

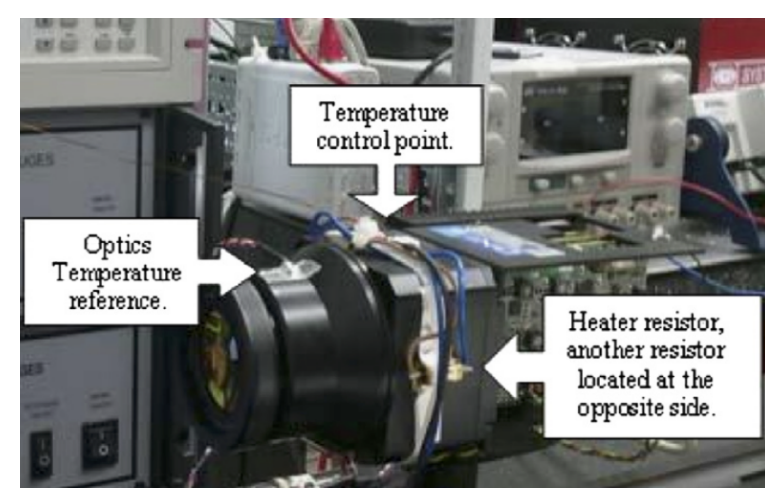

Figure 2: Scheme of the IRXCAM-640 camera module and SURNIA optics.

The filters defines two bands. The first band covers from $10.375 \mu \mathrm{m}$ to $11.225 \mu \mathrm{m}$, while the 
Table 3: Main characteristics of the SURNIA optics.

\begin{tabular}{c|c}
\hline Parameter & Value \\
\hline \hline Image diagonal & $21 \mathrm{~mm}$ \\
Stop size & $26.4 \mathrm{~mm}$ \\
Stop position & $22.9 \mathrm{~mm}$ \\
Back Working Distance (BWD) & $35.6 \mathrm{~mm}$ \\
Flange to Focal Plane (FP) & $39.4 \mathrm{~mm}$ \\
Focal length & $25 \mathrm{~mm}$ \\
F\# & 0.86 \\
Wavelength & $7-14 \mu \mathrm{s}$ \\
Circular FoV & $45^{\circ}$ \\
Transmission (typical) & $95 \%$ \\
Weight & $400 \mathrm{~g}$ \\
Min. Obj. Distance & $200 \mathrm{~mm}$ \\
Mount type & Threaded Mount \\
Focus type & Manual Focus \\
\hline
\end{tabular}

second band covers from $11.575 \mu \mathrm{m}$ to $12.425 \mu \mathrm{m}$ [10]. In terms of the FPA position, the first filter, which defines the first band, is located in the $\mathrm{X}$ axis from the pixel 1 to the pixel 320. The second filter, which defines the second band, is located from the pixel 321 to the pixel 640. Both filters covers the area located from pixel 1 to 480 in the $Y$ axis.

The system's FoV is $45^{\circ}$, enough to cover the area studied by the UV PDM of the EUSOBalloon, which is $12^{\circ}$ [11]. All the data taken by the IR camera, the temperature, humidity and pressure, is stored redundantly in two Solid State Devices (SSD). The whole IR camera system is placed inside an aluminium box, filled with nitrogen at a pressure of 1.5 bars, and covered by a styrofoam layer.

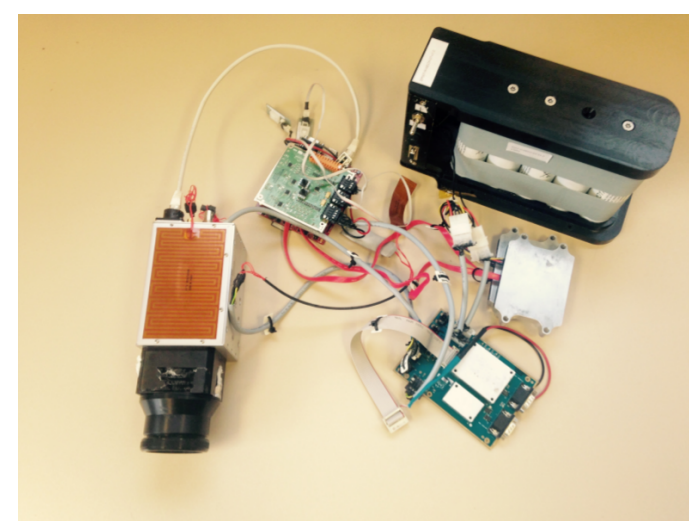

Figure 3: Some IR camera devices (current converter, SSD, IR camera module and the electronic).

The power supply of the camera consists of two rows of 5 cells. Every row has the dimensions: $208 \mathrm{~mm} \times 141 \mathrm{~mm} \times 84 \mathrm{~mm}$. Each cell weights $300 \mathrm{~g}$. The total capacity of the battery block is 


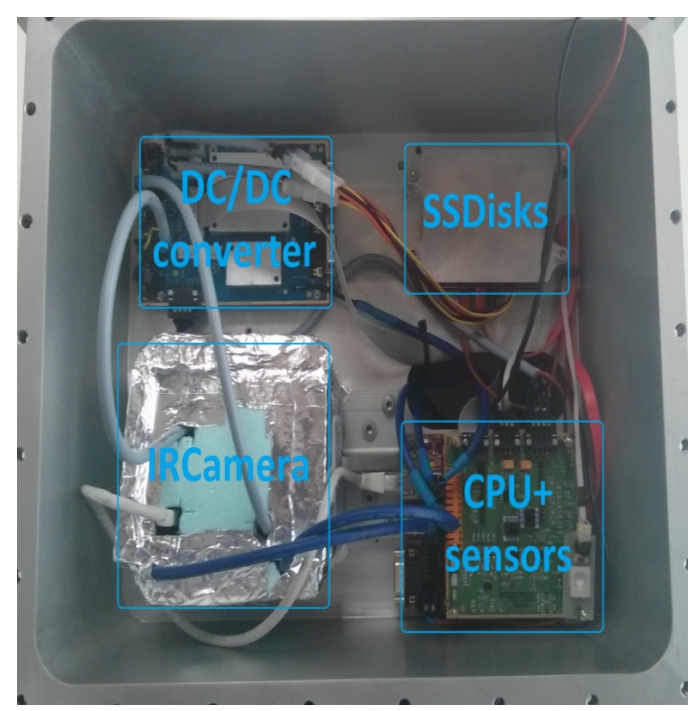

Figure 4: The battery pack together with all the IR camera devices.

$18 \mathrm{amp} \times$ hour, and gives an output voltage of $28 \mathrm{~V}$ DC.

Table 4: Power budget for all the IR camera instruments.

\begin{tabular}{|c|c|c|c|}
\hline & $\begin{array}{c}\text { Power } \\
(\mathrm{W})\end{array}$ & $\begin{array}{c}\text { Current } \\
(\mathrm{A})\end{array}$ & $\begin{array}{c}\text { Voltage } \\
(\mathrm{V})\end{array}$ \\
\hline CPU \& sensors & 4 & 0.15 & 5 \\
\hline IRcamera & 4.8 & 0.4 & 12 \\
\hline Heaters $(2 \times 4 \mathrm{~W})$ & 8 & 0.67 & 12 \\
\hline DC-DC $(12 \mathrm{~V})$ & 1.8 & 0.15 & 12 \\
\hline DC-DC $(5 \mathrm{~V})$ & 0.75 & 0.15 & 5 \\
\hline Total & 19.35 & - & - \\
\hline
\end{tabular}

\section{EUSO-Balloon IR Camera Data}

The EUSO-Balloon IR Camera took one picture every 80 seconds during the balloon flight held in August, 2014, over Timmins (Canada). It was functioning for around 17 hours, and therefore, took an overall of 753 photos. Although the EUSO-Balloon splashed down on a lake, the IR camera is water-proof, so all the data could be recovered. Due to the perfect isolation, the device is still perfectly working and the internal pressure only decreased to 1.3 bars. Moreover, after the flight the battery pack was still half charged.

Regarding the images, seven out of the 17 hours, the camera was functioning under water. Then, around 400 photos must be discarded from our analysis. If we take into account that the EUSO-Balloon conditions were not completely stable during the take off and landing, and we only consider for our study the photos taken during the proper flight (from around 03:30 to 8:20 UTC), 


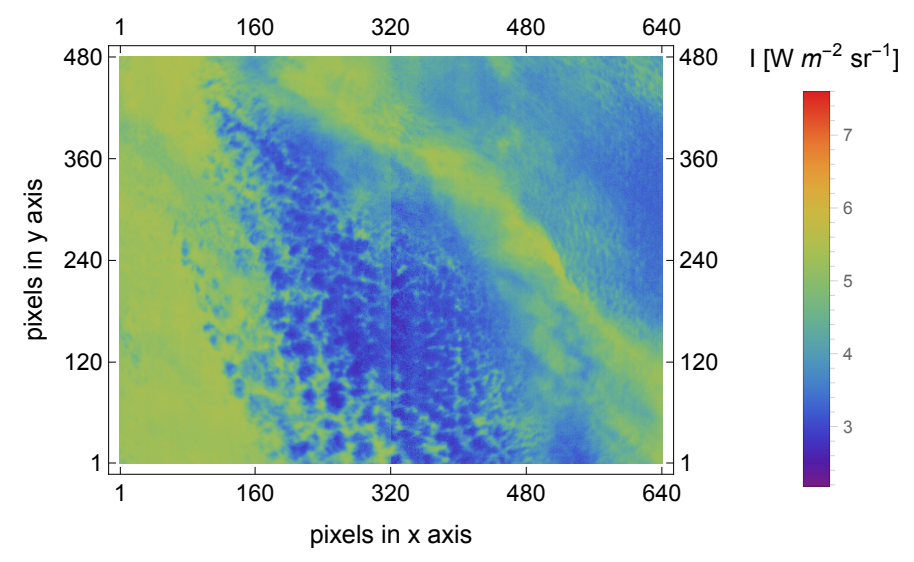

Figure 5: Image taken by the IR camera during the balloon flight.

220 photos need to be analyzed. Figure 5 is an example of the irradiance gotten from the IR camera data in one shot.

Once the analysis of the images is completed and brightness temperature is retrieved of IRCamera, the Cloud Top Height (CTH) is established using a Weather Research and Forecasting model [12]. Vertical profiles of temperature and humidity are obtained for different locations and at different times covering the whole of EUSO-Balloon track. Thus, an algorithm is built obtaining the cloud top height in each pixel of IR-Camera [13]. To assess the fit of the model firstly we have compared the vertical profiles of the WRF with adjacent radiosondes and subsequently, CTH retrieved by the algorithm is compared with those provided by other satellites flying over EUSOBalloon track. Also, some information related to the atmospheric optical depth can be obtained with the IR camera data [14].

\section{Acknowledgments}

This work is supported by the Spanish Government MICINN \& MINECO under projects AYA2009-06037-E/AYA, AYA-ESP 2010-19082, AYA2011-29489-C03-01 and AYA2011-29489-C03-02, AYA-ESP2012-39115-C03-01 and AYA-ESP2012-39115-C03-03, AYA-ESP 2013-47816-C4, MINECO/FEDER-UNAH13-4E-2741, CSD2009-00064 (Consolider MULTIDARK) and by Comunidad de Madrid under projects S2009/ESP-1496 \& S2013/ICE-2822. The calculations were performed using the Space and Astroparticle SPAS-UAH Cluster. J.A. Morales de los Ríos and H. Prieto want to acknowledge the financial support from the RIKEN-IPA program for one year research stay. M. D. Rodríguez Frías acknowledges the Swiss National Science Foundation (SNSF) for a Sabbatical research stay at the University of Geneva. L. del Peral acknowledges a senior grant for a Sabbatical stay at University of Geneva from the Spanish Ministerio de Educación, Cultura y Deporte under the "Salvador de Madariaga Programa Estatal de Promoción del Talento y su Empleabilidad en I+D+i, Subprograma Estatal de Movilidad del Plan Estatal de Investigación Científica y Técnica y de Innovación 2013-2016" M D Rodriguez Frias acknowledges a grant under the "Atraccio de Talent" program from the Vicerrectorado de Investigación de la Universidad de Valencia (Spain). 


\section{References}

[1] Adams, J.H. et al. (JEM-EUSO Collaboration). An evaluation of the exposure in nadir observation of the JEM- EUSO mission. Astropart.Phys., 44, pg 76-90, http://dx.doi.org/10.1016/j.astropartphys.2013.01.008, (2013).

[2] Rodríguez Frías, M. D. et al. for the JEM-EUSO Collaboration., The JEM-EUSO Space Mission: Frontier Astroparticle Physics at ZeV range from Space., Homage to the Discovery of Cosmic Rays. Nova Science Publishers, New York, ISBN: 978-1-62618-998-0, Inc, pg 201-212, (2013).

[3] The JEM-EUSO Collaboration, The JEM-EUSO Mission: Contributions to the ICRC 2013, Proc. of 33rd International Cosmic Ray Conference (ICRC), Rio de Janeiro, Brazil. ArXiv:1307.7071, (2013).

[4] Rodríguez Frías, M.D., Toscano, S., Bozzo, E., del Peral, L., Neronov, A., and Wada, S. and for the JEM-EUSO Collaboration., The Atmospheric Monitoring System of the JEM-EUSO Space Mission, Proceedings of the $2^{\text {nd }}$ AtmoHEAD Conference, Padova (Italy), ArXiv:1501.0482 [astro-ph.IM] (2014).

[5] Rodríguez Frías, M. D. et al. for the JEM-EUSO Collaboration., The Atmospheric Monitoring System of the JEM-EUSO Space Mission., Proc. International Symposium on Future Directions in UHECR Physics, The European Physical Journal, 53, 10005-pg1-7, (2013), http://dx.doi.org/10.1051/epjconf/20135310005.

[6] The JEM-EUSO Collaboration (corresponding authors: Toscano, S., Morales de los Ríos, J. A., Neronov, A., Rodríguez Frías M. D., \& Wada, S.)., The Atmospheric Monitoring System of the JEM-EUSO instrument., Experimental Astronomy, 37, (2014), doi=10.1007/s10686-014-9378-1.

[7] von Ballmoos et al. A balloon-borne prototype for demonstrating the concept of JEM-EUSO. Advance in Space Research. Vol. 53, pg: 1544-1560, (2014).

[8] The JEM-EUSO Collaboration (corresponding authors Morales de los Rios, J. A. \& Rodríguez Frías, M. D.), The infrared camera onboard JEM-EUSO, Experimental Astronomy, 37, doi=10.1007/s10686-014-9402-5, (2014).

[9] Rodríguez Frías, M. D. et al. for the JEM-EUSO Collaboration., Towards the Preliminary Design Review of the Infrared Camera of the JEM-EUSO Space Mission., Proc. International Cosmic Rays Conference, Rio de Janeiro, Brazil, arXiv:1307.7071v1 [astro-ph.IM], (2013).

[10] Morales de los Ríos, J. A., Joven, E., del Peral, L., Reyes, M., Licandro, J., and Rodríguez Frías, M. D., The Infrared Camera Prototype Characterization for the JEM-EUSO Space Mission., Nuclear Instruments and Methods NIMA, 749, 74-83, ISSN 0168-9002 (2014).

[11] Fernández Soriano et al. for the JEM-EUSO Collaboration. Performances of the Spanish Infrared Camera onboard the EUSO-BALLOON (CNES) flight on August 24, 2014. In this proceedings.

[12] Skamarock, W.C. and Dempsey, D. High-resolution winter-season NWP: Preliminary evaluation of the WRF and NMM models in the DWFE Forecast experiment AMS 17th Conference on NumericalWeather Predition,Washington D.C. Paper 16A.3 (2005).

[13] Merino, A.et al. for the JEM-EUSO Collaboration. Cloud Top Height estimation from WRF model: Application to the IR camera onboard the EUSO-Balloon (CNES). In this proceedings.

[14] Sáez-Cano, G. et al. for the JEM-EUSO Collaboration. Cloud Optical Depth obtained from the Spanish Infrared Camera data and the Laser shots of the USA helicopter during the EUSO-BALLOON (CNES) flight. In this proceedings. 